\title{
The Cusp Catastrophe Model of Major Accidents Based on Ideas of Black Swan and Its Application in Port Road Projects
}

\author{
Yanfeng Bai ${ }^{1, a^{*}}$, Ling Zhang ${ }^{1, b}$ and Xuejun Wen ${ }^{1, c}$ \\ ${ }^{1}$ Shanghai Municipal Engineering Design General Institute (Group) Co.Ltd, Shanghai, China \\ abaiyanfengwens@medi.com, bzhangling@smedi.com, cxuejun@smedi.com
}

Keywords: black swan, catastrophe theory, major accidents, cusp catastrophe model

\begin{abstract}
The normal accident prediction models can't predict the occurrence of major accidents very well, so the cusp catastrophe model of major accidents is set up on the base of ideas of black swan and catastrophe theory. In the model, the main factors of major accidents are regarded as the controls parameters and the functional status of the system as the state parameter. The model has filled up the blank field of studying emergency in the black area. It is well explained by the model that the project state depends on the interaction between human factors and material factors. In the further step, the mechanism of major accidents can be generalized. The model is applied in the case of port engineering. To avoid major accidents, the corresponding measures would be taken according to different states of the port engineering. Through the application, the model is proved to be useful in the engineering practice.
\end{abstract}

\section{Introduction}

The well-known events, such as "Basel", "Bhopal", "Mexico", etc., to promote the term "major accident" and "major accident control" generation, so that the control and prevention of major accidents around the world become an urgent issue. Current research on the prevention and control of major accident focused on the chemical, coal, transport, finance and other fields, while relatively little in the construction of large-scale civil engineering studies. 1980s, Wu using probability theory and decision theory applied to geotechnical engineering, foundation and slope studies on other security issues; Whitman underground engineering analysis calculated assessment of risk, risk assessment and gave underground engineering the basic processes and methods for the future to further develop risk-assessment model provided a theoretical basis and ideas; these results have important theoretical and practical significance for guiding geotechnical construction, but studies tend to ignore the influence of the human factor. Williams first proposed in 1958 that we must include the person's reliability in system reliability prediction; otherwise it will not represent the real situation of the system. 1972, Edwards use related hardware, software and environment between the four factors established SHEL conceptual model, the model is a milestone in the study of human error mode. Zhou Jian, Zhang Jiao, Tang Qun-yan etc. have taken the group to consider the impact of human factors in the excavation project, but the human factors need further study and complement.

Due to the occurrence of major accidents has burst, severity, urgency and other features, the use of predictive models in general, there will be a lag in response to mutation or mutations is not responding, making it difficult to achieve a better prediction. Based on the concept of Black Swan, combined with the mutation theory of a major accident factors into account people, objects and the environment, the establishment of a major accident cusp catastrophe model, uses the model to analyze the mechanism of large-scale projects of major accidents and made appropriate control measures.

\section{Catastrophe Theory}

Catastrophe theory is a mathematical theory of topological French mathematician Leineituomu raised in the late 1960s. The topological theory of dynamical systems designed to use to construct mathematical models of natural phenomena and social activities discontinuous change, the objective is 
to study the changes in the world of non-continuity of a sudden an emerging discipline. Potential catastrophe theory research is the system used to describe the potential function. Variable system potential function is divided into two categories: one is the internal variable behavior of the system variables or state variables, namely the system; the other is the control variable, namely the external variables of the system. Potential function mutation can be expressed as:

$$
\mathrm{V}=f\left(\varphi_{1}, \varphi_{2}\right)
$$

Where the state $\varphi_{1}$ is a collection of system parameters $x, \varphi_{1}=\left\{x_{1}, x_{2} \cdots x_{n}\right\} ; \varphi_{2}$ is a collection of system control parameters $u, \varphi_{2}=\left\{u_{1}, u_{2} \cdots u_{n}\right\}$.

When the control variable is the number of no more than 4 , then only seven potential function $\mathrm{V}$ forms, which cusp catastrophe is the most widely applied one of the most intuitive function is only two control parameters and a state parameters mutant structure, its potential function as follows:

$$
V(x)=x^{4}+u_{1} x^{2}+u_{2} x
$$

Where $x$ is the state variable, $u_{1}$ and $u_{2}$ are the control variable, the graphic shown in Fig.1. The cusp catastrophe manifold looks like a fight wrinkles cloth surface consists of three layers, can be divided into upper, middle and lower clover. The upper leaf surface, the leaves are smooth, so is stable. Leaves a functional state of the system is good; while the system is under the surface leaves poor functional status. In part outside the crease, from the initial to the end state is a gradual process of system functions; And for that part of the mid-crease in the curved edge of the folded, functional state of the system will be mutated, it can be dropped directly from the leaf surface under leaves, or leaves from the lower lobe Jump directly, rather than through the middle, showing the state of instability, namely mutations.

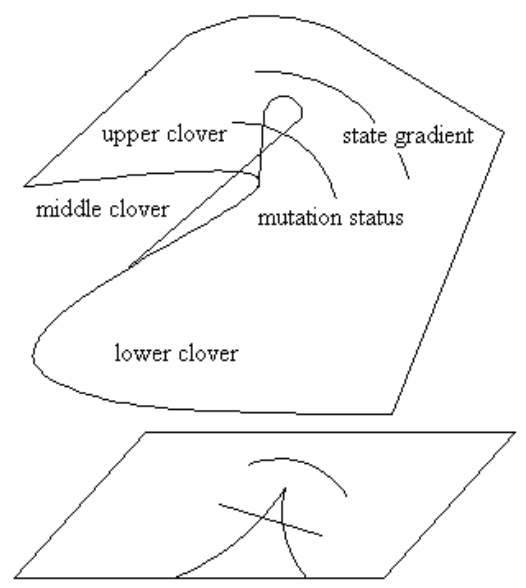

Fig.1 Manifold mutation and bifurcation set of cusp catastrophe

\section{Major Accidents Cusp Catastrophe Model}

1. Factors causing a major accident

Many factors cause a major accident, summed up into human factors, physical factors and environmental factors. The human factor is mainly caused by human subjectivity, including human misjudgment, error analysis and error behavior and willingness to change and defect management. Factor is the hidden objects in the object itself impending major accident some inherent insecurity. External causes of environmental factors belong to the system refers to the system caused by a mutation in the external environment, including the natural environment system and social environment in two ways.

These three factors often involves both the natural and social sciences, has great randomness, so a major accident should fall within the purview of the black area. According to the concept of black swans found that due to the limitations of the people of knowledge, the researchers conducted a major accident is impossible to accurately predict; But we can according to their asymmetry, using the conventional system knowledge to prevent and control major accidents.

2. Cusp catastrophe model 
1) Cusp catastrophe model building

Major accident is a typical negative black swan event; we can establish a model to analyze the mechanism by which they are incurred. Environmental factors such as major accidents classified as among the factors that matter, can be seen as the result of a major accident factors and human factors matter the role. Now the human factor $\mathrm{H}, \mathrm{M}$ factors matter as two control variables, the functional state of the system $\mathrm{F}$ as a state variable, the cusp catastrophe model potential function is:

$$
V(H, M, F)=\frac{1}{4} F^{4}+\frac{H}{2} F^{2}+M F
$$

It mutation manifold equation:

Bifurcation set equation is:

$$
F^{3}+H F+M=0
$$

$$
4 H^{3}+27 M^{2}=0
$$

Mutation manifold surfaces and bifurcation point of the model set shown in Fig. 2, the curve of the figure shows the functional status of $\mathrm{F}$ factor with the human factor $\mathrm{H}$ or $\mathrm{M}$ matter changes in the process of change. Analyzed as follows:

(1)For the curves $a_{1} b_{1} c_{1} d_{1}$, curve downward on the leaf from leaf development, without fold lines, indicating that even if the system is in constant state of deterioration, but no major accidents.

(2) For the curves $a_{3} b_{3} c_{3} d_{3}$, from $b_{3} \rightarrow c_{3}$ status $F$ jumps occur, it changes the value, that is $\Delta F_{3}=F\left(H_{b 3}, M_{b 3}\right)-F\left(H_{c 3}, M_{c 3}\right)$, from $\mathrm{b}_{3} \rightarrow \mathrm{c}_{3}$ have a major accident. On the bifurcation set, the corresponding curve is a curve through the points of disagreement set two edges; Similarly, the curves $a_{2} b_{2} c_{2} d_{2}$ and $a_{4} b_{4} c_{4} d_{4}$ respectively $b_{2} \rightarrow c_{2}$ and $b_{4} \rightarrow c_{4}$ jumps occur, the size of the sudden jump and value respectively $\Delta F_{2}$ and $\Delta F_{4}$.

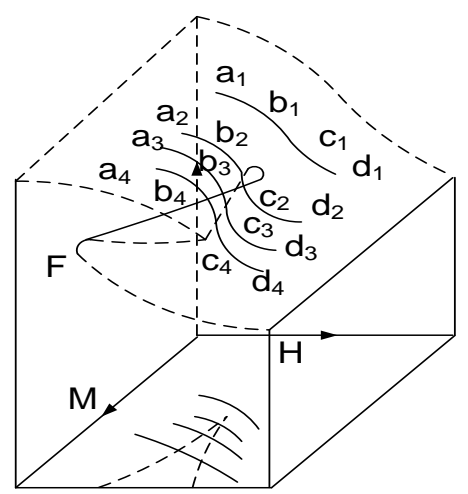

Fig.2 Cusp catastrophe model of major accidents

(3) Comparison shows that the value of three mutations $\Delta F_{4}>\Delta F_{3}>\Delta F_{2}$, suggesting that the former is a large major accident, in the middle of a major accident for the medium, which is small compared to a major accident, is reflected in the focus points of disagreement, compared with three different length curve.

Through qualitative analysis, changes in the status of the system function parameter depends on changes in the two control parameters, control parameters determine whether the size of the state parameter jumps and jumps values occurs, the size of the sudden jump reflects the value of major accident hazards degree.

2) Application of cusp catastrophe model

Shown in Fig. 3 cusp mutation model, there are a, b, c, d, e, f, g, h, 8 points, and the functional state of the relationship between the points as follows:

(1) If you keep the human factor $\mathrm{H}$ unchanged, but the work environment, equipment and other factors gradually deteriorated, namely $\mathrm{M}$ increases, this will inevitably affect the quality of the works on the model path $\mathrm{a} \rightarrow \mathrm{b}$. Conversely, if things remain constant factor $\mathrm{M}$, and gradually increased human error, that $\mathrm{H}$ increases, which also makes poor quality projects, on the model corresponding path for $\mathrm{a} \rightarrow \mathrm{f}$. 
(2)If the factor human factor $\mathrm{H}$ and $\mathrm{M}$ while larger objects, means the external work environment, machinery and equipment, construction quality and other factors gradually deteriorated, and human error are increasing, so is bound to a major accident in the model path $\mathrm{a} \rightarrow \mathrm{c} \rightarrow \mathrm{d}$.

(3) If $\mathrm{M}$ and $\mathrm{H}$ stand at a large value, such as e-point, $\mathrm{i} \rightarrow \mathrm{e}$ the external environment, machinery and equipment in poor condition but also a lot of human error, only to reduce the human factor, and not the poor have been built the demolition and reconstruction or rectification works, does not improve the working environment, so this does not improve the overall quality of the project, but also can lead to a serious accident on the model path $\mathrm{e} \rightarrow \mathrm{g} \rightarrow \mathrm{h}$.

(4) If the $\mathrm{M}$ and $\mathrm{H}$ stand at a large value, such as e same point can be the first to reduce the value of $\mathrm{M}$, then lower the value of $\mathrm{H}$, which is the first built by the rectification works to improve the quality of follow-up projects to improve the working environment measures to reduce the impact of physical factors, and then improve the quality and reduce the impact of human factors, so you can avoid fold lines, improve the quality of works under the premise of no major accidents, the path on the model corresponding to $\mathrm{e} \rightarrow \mathrm{f} \rightarrow \mathrm{a}$.

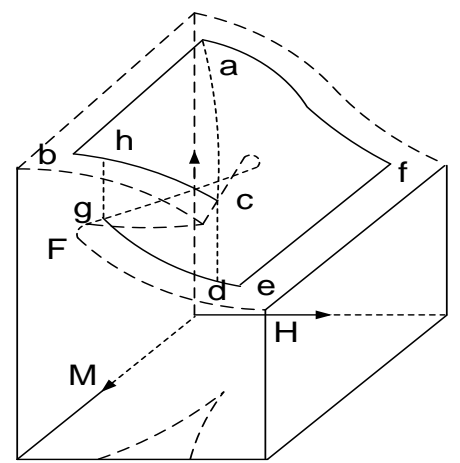

Fig.3 Cusp catastrophe model of major accidents

(5) By the catastrophe model shows that the system state occurs from point $\mathrm{c}$ to $\mathrm{d}$ sport when mutation is:

$$
\Delta F=\left|F_{b}-F_{c}\right|=(-3 H)^{\frac{1}{2}}=\left(\frac{27}{2} M\right)^{\frac{1}{3}}
$$

From Equation (6) shows that and the sudden jump of the system functions is proportional visible with $(-3 H)^{\frac{1}{2}}$ or $\left(\frac{27}{2} M\right)^{\frac{1}{3}}$,human factors influencing factors greater than the substance of the system functions.

This concludes this part shows that the mechanism may be a reasonable explanation of the model generated by a major accident; while a major accident as a negative black swan events, in order to avoid the impact of our work should take measures in accordance with the conservative analysis model, and to ensure the implementation of measures to reduce the negative factors in particular human factors.

\section{Projects}

\section{Project Overview}

The road is being constructed in a harbor in the new container Deepwater, with 15.9 meters depth container hub port. West Port uses its land reclamation to form sand into land elevation of $+8.0 \mathrm{~m}$, the total volume of about $31,020,000 \mathrm{~m}^{3}$, and backfilling total area ofabout $2,100,000 \mathrm{~m}^{2}$. The maximum thickness of the filling is over $38 \mathrm{~m}$, and the average filling thickness is $15 \mathrm{~m}$. Using land-based plastic drainage plate inserted to play plus preloading method for reinforcement. The road tide surface and the design of the road height used the Xiaoyangshan theoretical lowest ebb face as the starting surface. The relationships between Xiaoyangshan station each datum shown in Fig. 4.

2. Natural environmental conditions 
The port area of land formed by weather conditions, such as the effects of temperature, precipitation, wind conditions, tropical cyclones, cold and other factors, while the affected hydrological conditions, such as the tidal waters of moderate intensity, the average tidal range of $2.75 \mathrm{~m}$; trend was typical reciprocating movement of the flow patterns, the trend of the maximum possible flow rate $1.60 \mathrm{~m} / \mathrm{s}$; proposed project waters to storm the main frequency of small swell, wave height / period generally are small.

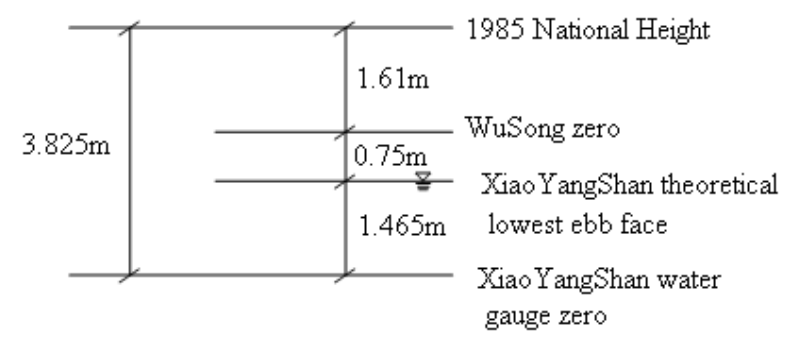

Fig.4 Relationship among the base levels

3. Cusp catastrophe model in the project

Port road projects including the formation of embankments and hydraulic fill sand and other construction processes, equally affected people, physical and environmental factors, with the cusp catastrophe model for analysis of the project, additional analysis as a routine risk.

Analysis of survey data, the engineering foundation landslides or deformation occurs port road is the main reason for poor soil conditions, is caused by excessive hydraulic fill sand foundation overall landslide important reasons. When the original ground elevation below the lowest point position, the accident occurred at the lowest point of time; when the original ground elevation above the original lowest ebb face, the accident occurred in the tide of time below the original ground level. So, after the sand pile foundation soil conditions remain poor handling, hydraulic fill sand too fast, tidal influence, the overall decline is a major factor causing the foundation of the main objects of the accident, and limited understanding of the level of on-site management personnel, lack of management efforts human factors such as human error is the cause of major accidents. According to the above empirical knowledge, application cusp catastrophe model can predict several forms of serious accidents that may occur in this project and propose appropriate measures.

1) Fig. 3 shows the path is an accident prone $a \rightarrow c \rightarrow d$, description of tidal effects due to lack of awareness, poor quality of construction of embankment and foundation, coupled with excessive personnel during the construction of human error, which will lead to roadbed landslides and other major accidents. Therefore, during the construction of the road construction should strengthen management, improve the construction side, the level of awareness Supervisor to monitor side and the construction side and other related personnel to deepen their understanding of the importance of quality engineering, construction process, thereby reducing the impact of human factors . Site construction personnel earnestly urge foundation reinforcement construction process to ensure that the replacement rate and depth of the sand pile to meet the design requirements to ensure the strength of the soil up to the design requirements; supervise construction personnel in strict accordance with the design and construction specifications for construction, strict control of Reclamation rate; strictly controlled loading rate, the implementation of block loading, strengthen timely monitoring, should grasp the ground deformation and consolidation, once the abnormal situation, immediately stop the load and take the appropriate measures; simultaneously pay close attention to changes in water level difference; to this reduces the impact of factors matter.

2) Fig.3shows the path $e \rightarrow g \rightarrow h$ mutations may also occur, indicating that in the case of the construction section has serious quality problems of port road projects, not only emphasized the construction process in the future to improve the only man-made factors, reduce human error, the same should also be of serious quality problems for rectification reinforced part of the project, where necessary, rework the reconstruction cannot be planted quality problems. 
3) From Fig.3 $e \rightarrow f \rightarrow a$ road map shows, in the case of emergency port road projects, the physical descendants should also improve construction quality is the first, and then strengthens the training and education of personnel in order to reduce human error, so as to reduce the risk of a major accident.

\section{Conclusions}

(1)The prediction model based on generally can not elaborate mechanism of defect major accident, combined with a sharp point mutations characteristic functions and major accidents, the establishment of a major accident cusp catastrophe model fill the black area in the field of emergency blank; while according to black Swan philosophy, we cannot expect the application of the model to make accurate predictions of major accidents, but the model can be applied to reduce the probability of a major accident.

(2) In this model, the project is a system of two control parameters and state parameters, which are people and objects. The result of the good and bad of human factors and physical factors of the combined effect of the functional status of the system; according to black Swan philosophy, there are likely to be affected negatively when the black swan event, we should take a very cautious and conservative measures, and in the model to minimize the impact of two factors and note the occurrence of small probability events.

(3) The use of the model can guide the construction of road projects Port: (1) during road construction, the tidal effects of poor construction quality, more human error under the circumstances, would lead to a major accident; (2) construction in serious quality problems, some projects have to deal with the construction or reconstruction of rectification, not trusting to luck; while strengthening the management of human factors, in particular, to reduce the impact of two factors on subsequent human factors engineering; (3) when major incidents occur, should improve the quality of the project, and then minus factors villain.

\section{References}

[1] Fell R., Landslide risk assessment and allowable risk, Canadian Geotechnical Journal, 31(1994) 261-271.

[2] Jones D D, Walters CJ, Catastrophe theory and fisheries regulation, Journal of the Fisheries Research Board of Canada, 12(1976) 829-833.

[3] Ling Fuhua, Catastrophe Theory and Its Applications Shanghai, Shanghai Jiaotong University Press,Shanghai, 1987.

[4] Loehle C., Optimal stocking for semi-desert range: a catastrophe theory model, Ecological Modelling, 27(1985)285-297.

[5]Nilsen B., Models and model uncertainty in the context of risk analysis, Reliability Engineering and System Safety, 79(2003)309-317.

[6]Whitman R V., Evaluating calculated risk in geotechnical engineering, Journal of Geotechnical Engineering, ASCE, 110(1984)145-188.

[7]Williams H L., Reliability evaluation of the human component in man-machine system, Electrical Manufacturing, 4 (1958)78-82. 